\title{
Prediction of Crop and Weed Growth Stages using Neural Network in Machine Learning
}

\author{
Devansh Dutt, M. Jasmine Pemeena Priyadarsini, Smruti Ranjan Khuntia
}

\begin{abstract}
This paper presents the structure, usage and assessment of a convolutional neural system-based methodology has been applied for the arrangement of various phenological phases of plants. Our CNN design can consequently characterize distinctive phenological phases of eleven sorts of plants. So as to assess the presentation and productivity of our profound learning-based methodology, an old-style AI approach dependent on physically removed highlights is additionally executed. Textural highlights dependent on GLCM highlights have been removed and joined arrangement of highlights are taken care of into an AI calculation. The arrangement pace of the methodology dependent on physically extricated highlights is contrasted with those of our CNN based methodology. Trial results demonstrate that CNN put together methodology is fundamentally powerful with respect to the eleven sorts of plants we investigated. There are a wide range of ways profound learning can be applied on a dataset relying upon the size of the dataset. There are many research headings that we are intending to take for arranging phenological stages. Future work may comprise of building our own CNN design without any preparation especially prepared for arranging phenological phases of plants, just as trying different things with other pre-prepared CNN models and making sense of an approach to recognize infections in crops.

Keywords: Phenological Stages, Crop and Weed Growth Stages, Neural Network, Machine Learning
\end{abstract}

\section{INTRODUCTION}

Assurance of the phenological phases of plants is crucial for the extension of solid and beneficial plants. The information on progress times of phenological phases of a plant can give important information to arranging, sorting out and auspicious execution of rural exercises (showering, water system and so on). Past works center around either just about plant distinguishing proof or just phenological stage acknowledgment utilizing just a single surface examination technique. Our way to deal with the issue is novel on the grounds that not just the prominence of the plant type or the ubiquity of just the phenological stage, yet additionally joint recognizable proof of the plant type and the phenological stages are given a few surface and shading highlight

Revised Manuscript Received on June 10, 2020.

* Correspondence Author

Smruti Ranjan Khuntia*, Electronics and Communication Engineering, Vellore Institute of Technology, Vellore, India. E-mail: smrutiranjan.khuntia2016@vitstudent.ac.in

M. Jasmin Pemeena Priyadarshini, Electronics and Communication, Vellore Institute of Technology, Vellore, India. E-mail: jasmin@vit.ac.in

Devansh Dutt, BTech in Electronics and Communication Engineering, Vellore Institute of Technology, Vellore, India. E-mail: smrutiranjan.khuntia2016@vitstudent.ac.in

(C) The Authors. Published by Blue Eyes Intelligence Engineering and Sciences Publication (BEIESP). This is an open access article under the CC BY-NC-ND license (http://creativecommons.org/licenses/by-nc-nd/4.0/) examination strategies. In this work, an investigation is led to think about the utilization of a few picture surface highlights for the arrangement of the plants and their phenological stages. Checking phenology of horticultural plants might be a basic comprehension in exactness farming. Essential enhancements are regularly accomplished with exact recognition of phenological change of plants which may hereafter improve the planning for the gather, bug control, yield expectation, ranch observing, catastrophe cautioning and so forth. Numerous nations over the world have been creating activities to manufacture national farming observing system frameworks, since inducing the phenological data adds to a superior comprehension of connections between efficiency, vegetation wellbeing and ecological conditions. In this paper, we use a profound learning engineering to perceive and characterize phenological phases of a few sorts of plants $\mathrm{A}$ pre-prepared Convolutional Neural Network design (CNN) is utilized to consequently extricate the highlights of pictures.

Farmers not just need to battle for the better yield against the catastrophic events yet additionally need to handle the misfortunes of the net yield as a result of land treatment particulars and untalented work as well. In case of insufficient utilities and assets, despite capricious emergencies, their benefit openings and work are relatively and unfavorably influenced. Notwithstanding, in this period of innovation, the situation may get changed as the Information and Communication and related fields of innovation are giving an incredible to such sort of emergency dealing with. Here in this paper, the strategy which might be utilized to contrast the harvest leaf shading and the stage forecast has been proposed for getting an insight concerning the necessity of plant, before enough to get the yield utilizing profound learning calculation.

\section{PROPOSED WORK}

The term automated picture suggests getting ready of a two-dimensional picture by a PC. In a progressively broad setting, it gathers propelled treatment of any two-dimensional data. A modernized picture is an assortment of veritable or complex numbers addressed by a set number of bits. An image given inside the sort of a straightforwardness, slide, photograph or a X-bar is first digitized and taken care of as a system of twofold digits in memory. This digitized picture would then have the option to be set up just as appeared on a significant standards TV screen. For appear, the image is taken care of during a fast access pad memory, which resuscitates the screen at a pace of 25 housings for consistently to deftly an apparently relentless introduction. In this idea the phases of development are essential to assist ranchers with improving the yield.

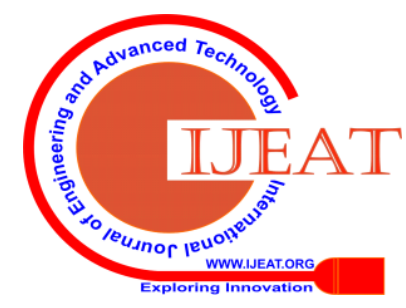




\section{Prediction of Crop and Weed Growth Stages using Neural Network in Machine Learning}

Data on which weed species are available inside rural fields is significant for site explicit weed the executives. This paper presents a strategy that is equipped for perceiving plant species in shading pictures by utilizing a convolutional neural system. In light of GLCM, division and arrangement can be handily performed. Consequently time utilization will be less. SVMs are a lot quicker than multilayer perceptron systems and precisely anticipate target likelihood score. The objective is to design and implement a convolutional neural network-based approach has been applied for the classification of different phenological stages of plants. The existing system had the following drawbacks [1] In the existing there was no proper system for preventing diseases in plants. [2] They manually check for the health conditions of the plants. [3] Prediction of diseases is tough for farmers as new type of bacterial and viral diseases keep coming up. Image processing can reduce the total information of plant image to a manageable amount, by increasing edges and making geometrical corrections, before the analysis of measurements and identification of some specific details like size, area and shape. The biggest advantage of image analysis is that it can view specific areas and contrast colors. This allows visual explication and interactive analysis by the computer. The analysed images can also be stored in a mass memory. Thus, plant leaf area is often wont to predict the expansion of plants as functions of environmental conditions. Disruptive harvesting of plants by sampling of leaves is the most accurate way to measure the leaf area, but it is not good to make repeated measurements on the same plants or remote measurement of plants. Moreover, the worlds of the leaves are often predicted supported the correlations between the area and dimensions of leaf size and shape.

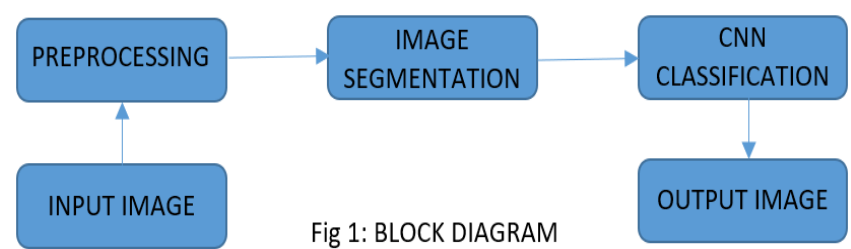

The proposed system consists of four major modules

[1] Pre-processing: The images which are taken as input may have some possibility of disturbances like noise, blurriness etc. The main aim of image pre-processing is to improve image data and to remove these unnecessary things for making suitable for our process. Image pre-processing is used to correct degradation in the input images. Pre-processing techniques involves enhancing contrast, removing noise, etc.

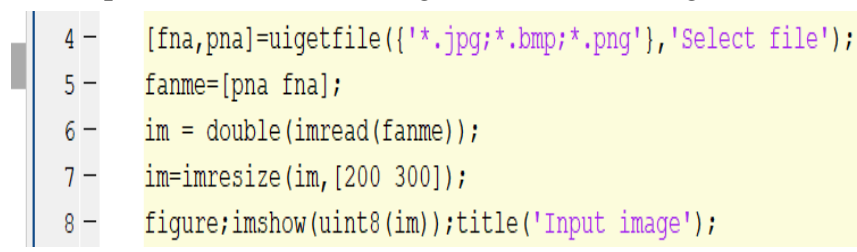

We take input from the user and store it into file name and path name, Next, we take the image in double format and resizing it for easy pre-processing. Next, converting it into uint8 format and displaying the input image. Here, we take a Maize plant for testing purpose Plant for testing purpose.

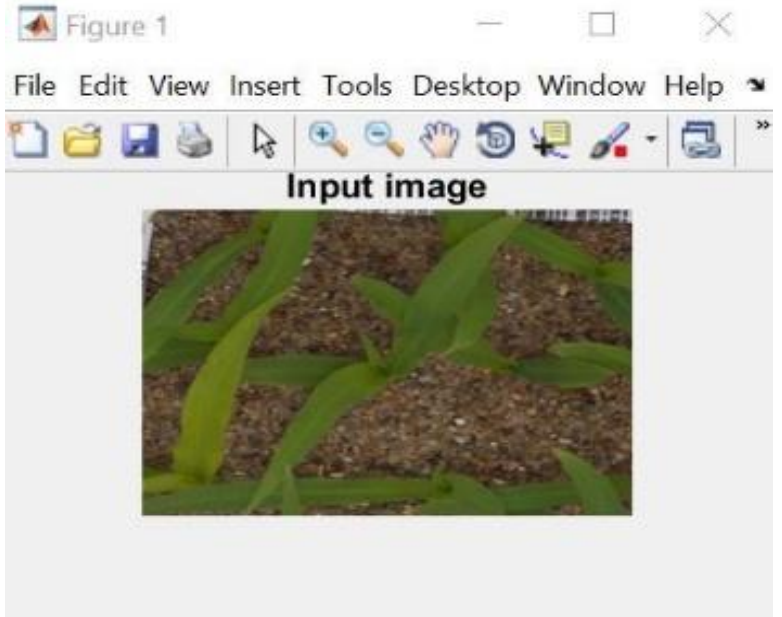

Fig 2: Input Image

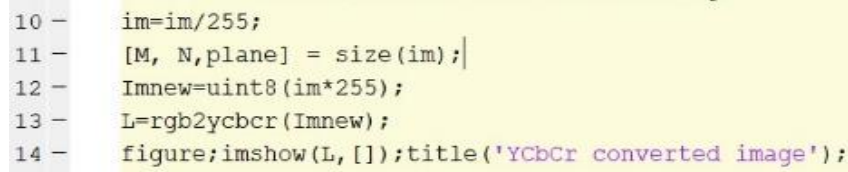

Next, we divide the image by 255 to describe the range from 0 to 255. Black is denoted as 0 and White is denoted as 255 . Values in between represent different shades of gray. Next, we classify the image into $\mathrm{M}$ rows, $\mathrm{N}$ columns and plane stores the RGB layers of the image. Now we convert the image into $\mathrm{Y}, \mathrm{Cb}, \mathrm{Cr}$ format. $\mathrm{Y}$ represents luma component and $\mathrm{CB}$ and $\mathrm{CR}$ represent the blue-difference and red-difference chroma components.

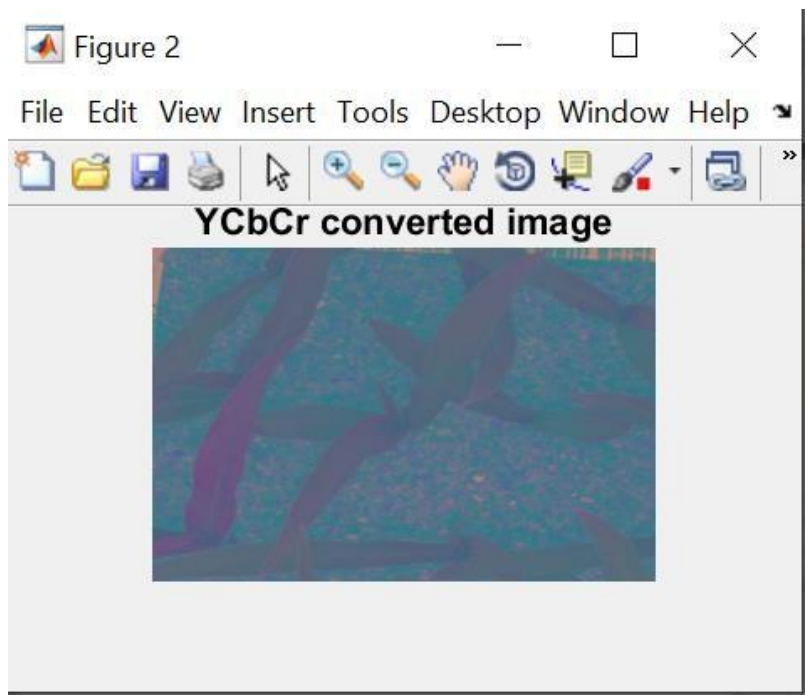

Fig 3: YCbCr Converted Image

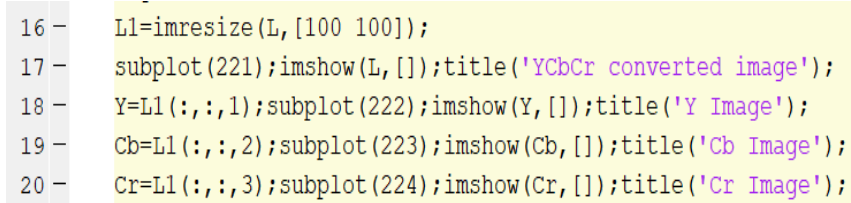

Next, we classify individual $\mathrm{Y}, \mathrm{Cb}, \mathrm{Cr}$ component individually. The individual $\mathrm{Y}, \mathrm{Cb}, \mathrm{Cr}$ component we get is-

Published By:

Blue Eyes Intelligence Engineering \& Sciences Publication

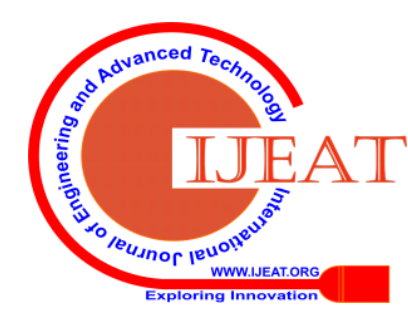




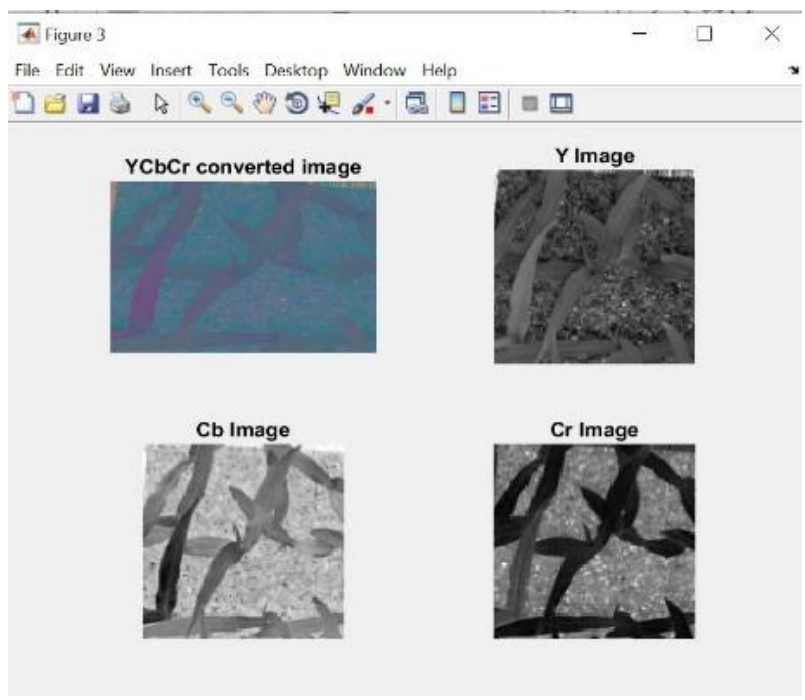

Fig 4 Y, Cb, Cr Components

[2] Segmentation: Segmentation is to isolate the homogeneous zone. Many mechanized procedures were proposed to defeat the repetitive and tedious undertaking of human specialists in tallying and grouping phases of plant. We resize the image after conversion. We convert the $\mathrm{Cb}$ and Cr component into double format for calculation purpose and create a Gaussian Distribution.

The reason for this stage is to section the leaf picture from the relative foundation. The separated $\mathrm{Cb}$ and $\mathrm{Cr}$ coefficients from our preparation pictures during pre-handling stage are currently used to construct a Gaussian Distribution as appeared in conditions 1, 2, 3, 4.

bmean $=$ mean $(\mathrm{cb})(1)$

Where $\mathrm{cb}$ is that the row vector containing all $\mathrm{Cb}$ coefficients obtained from our training images, and bmean is that the blue mean of this vector.

$$
\text { rmean }=\text { mean }(\mathrm{cr})
$$

Where $\mathrm{cr}$ is that the row vector containing all $\mathrm{Cb}$ coefficients obtained from our training images, and rmean is that the red mean of this vector.

$$
\text { brcov }=\operatorname{cov}(\mathrm{cb}, \mathrm{cr})(3)
$$

(Where brcov is that the co-variance of the 2 row vectors $\mathrm{cb}$ and cr. The result's a 2x2 matrix)

$\operatorname{mag} \operatorname{Cov}=(\operatorname{brcov}(1,1) * \operatorname{brcov}(2,2)-\operatorname{brcov}(2,1) * \operatorname{brcov}(1,2))$

Where magCov is the magnitude of the brcov.

In the testing stage the Gaussian distribution is applied on the info test picture to achieve the division stage as appeared in underneath conditions.

$$
\mathrm{x}=[(\mathrm{cb}-\mathrm{bmean}),(\mathrm{cr}-\text { rmean })]
$$

Gaussian circulation is applied to check pictures inside the YCbCr space to extricate our significant pixels that are most likely included to our districts of intrigue ROI.

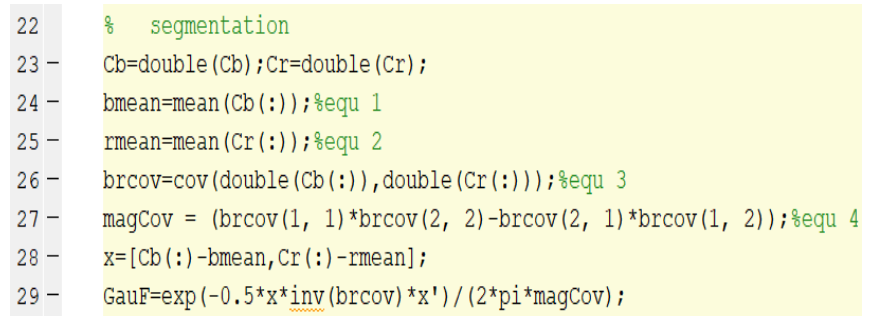

[2.1] Normalization: Here, we reshape the mean of our Gaussian Function using the size vector of Cb-by-1 and

Blue Eyes Intelligence Engineering \& Sciences Publication
Published By:

(C) Copyright: All rights reserved.

Cb-by-2 matrix. Next, we take absolute value of the resized image and deploy it back to our range of 0- 255 and convert it back to uint8 format.

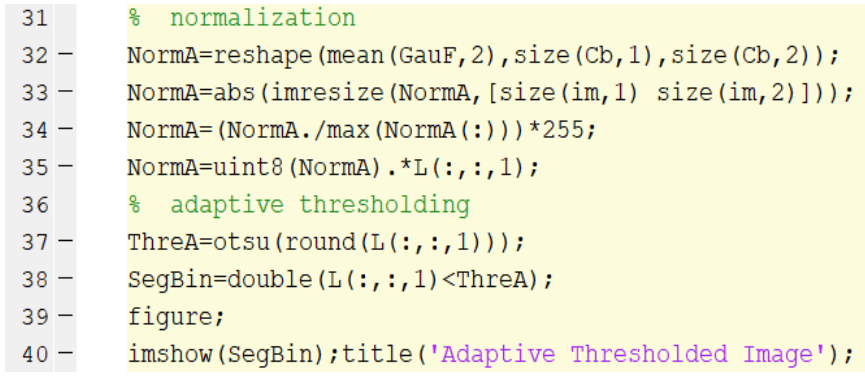

[2.3] Adaptive Thresholding using Otsu's Method: In the simplest form, the algorithm returns a single intensity threshold that separate pixels into two classes, foreground and background. SegBin returns a Binary image Whose value is less than the Threshold value found by otsu method.

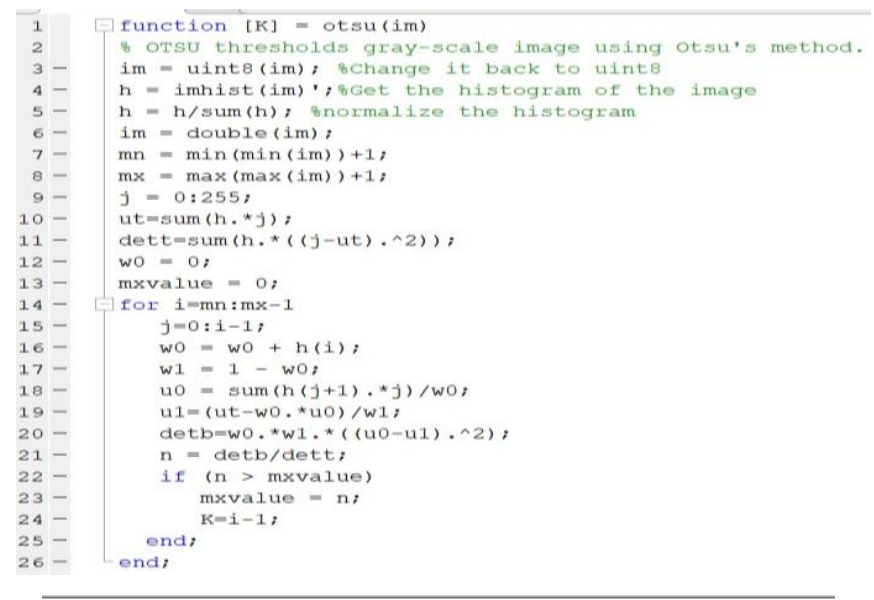

Figure 4

File Edit View Insert Tools Desktop Window Help $\mathbf{x}$

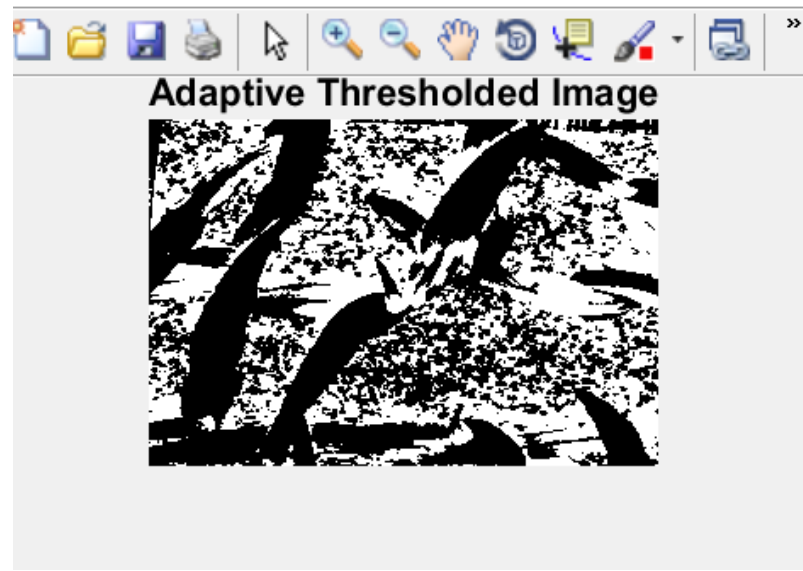

Fig 5: Adaptive Threshold Image

[2.4] Masking: We create a im-by-im matrix of zeros and store it in ReYcbcr and ReRGB. Next, we run a for loop for $\mathrm{i}=$ $1: \mathrm{M}$ and $\mathrm{j}=1: \mathrm{N}$ i.e. for every element in row and column of our image. If the SegBin for that particular matrix element is equal to 1 then we store the double value of $\mathrm{YCbCr}$ component into ReYcbcr matrix and normal RGB value to ReRGB.

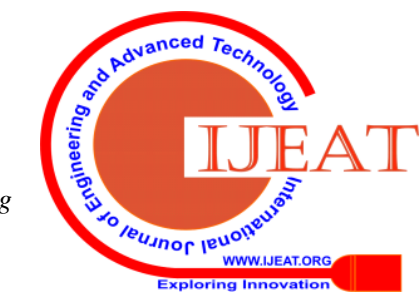




\section{Prediction of Crop and Weed Growth Stages using Neural Network in Machine Learning}

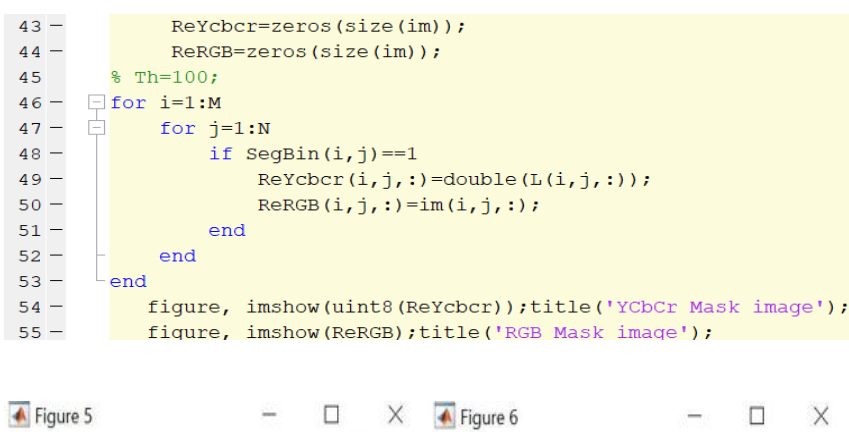

File Edit View Insert Tools Desktop Window Help $\boldsymbol{x}$ File Edit View Insert Tools Desktop Window Help $\boldsymbol{v}$

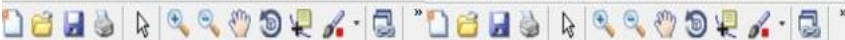
YCbCr Mask image

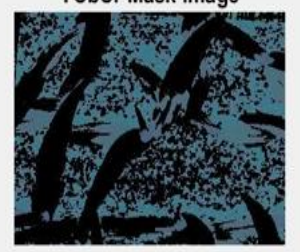

RGB Mask image

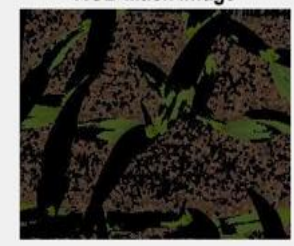

Fig 6: YCbCr and RGB Mask Image

[2.4] K-mean Clustering: Clustering looks the gathering of a multi-dimensional informational collection by contrasting likeness or disparity esteems. Here we use technique for partitional bunching, parcel the given examples has K-groups, the highlights in a specific example bunch are fundamentally the same as each other than to those highlights in various example gatherings. It is computationally extremely productive, if the bunches are very much isolated and conservative this technique gives agreeable outcome. For segment the powers saw in the picture into comparative gatherings they use picture division by utilizing bunching technique and divided picture into groups or locales.

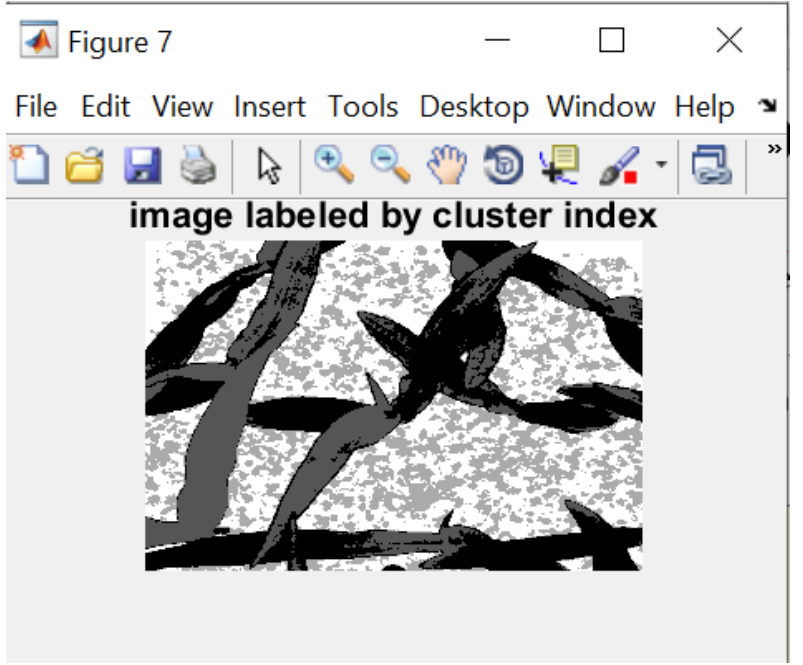

Fig 7: Image Labeled by Cluster Index

We create a 1-by-3 empty cell array and store it as segmented_images. We create an array containing [1,1,3] copies of pixel_labels in the row and column dimensions. The size of rgb_label is size(pixel_labels)*[1,1,3] when pixel labels is a matrix. We run a for loop for 1: n Colors(=4) and extract the Contrast Enhanced Image and all the 4 clusters.

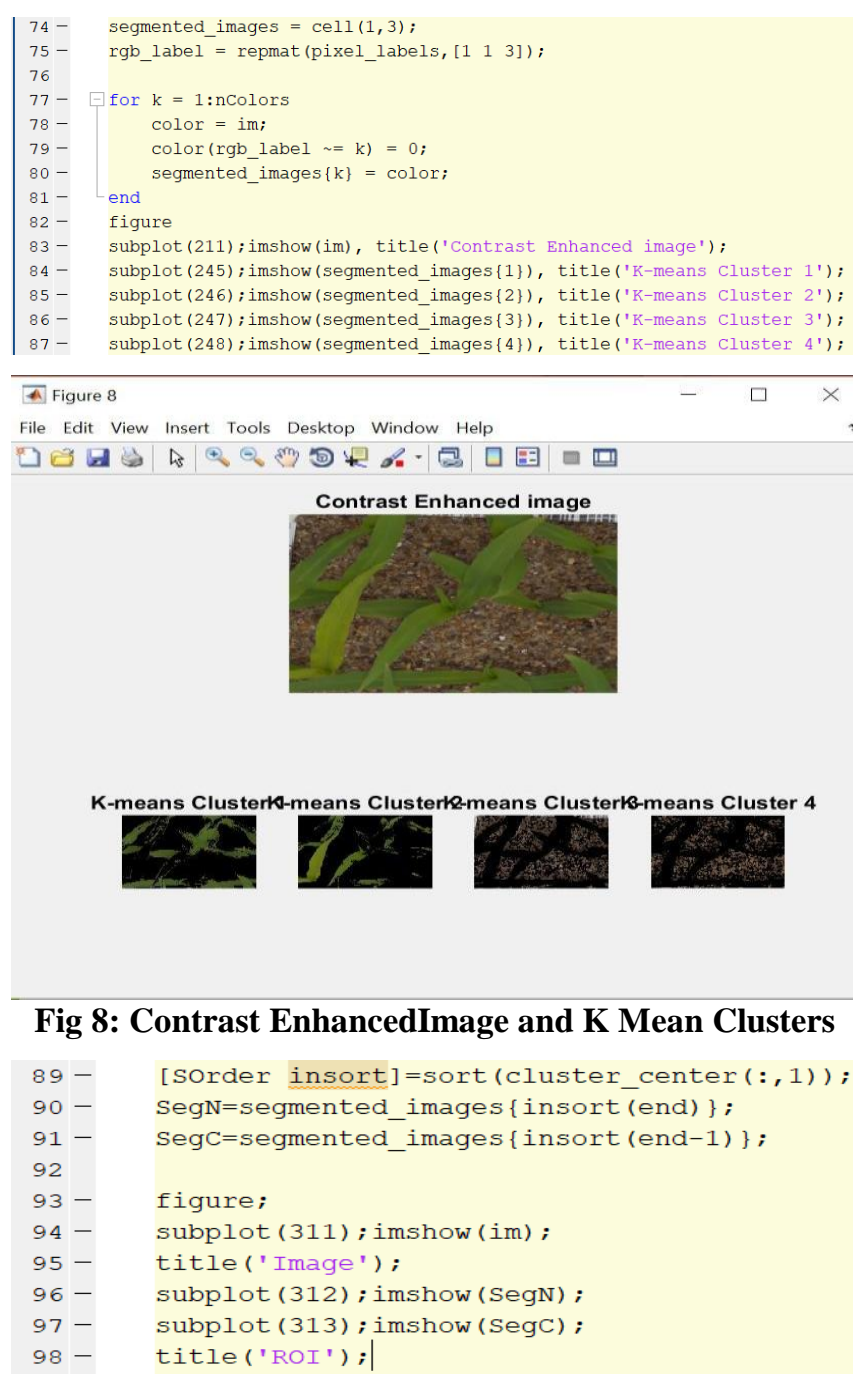

SOrder lists the sorted data and insort contains the corresponding indices of cluster_center, Next, we store the the last and last but one indice in SegN and SegC respectively, Next, we display the last 2 indices, where last but one indice is our Region of Interest

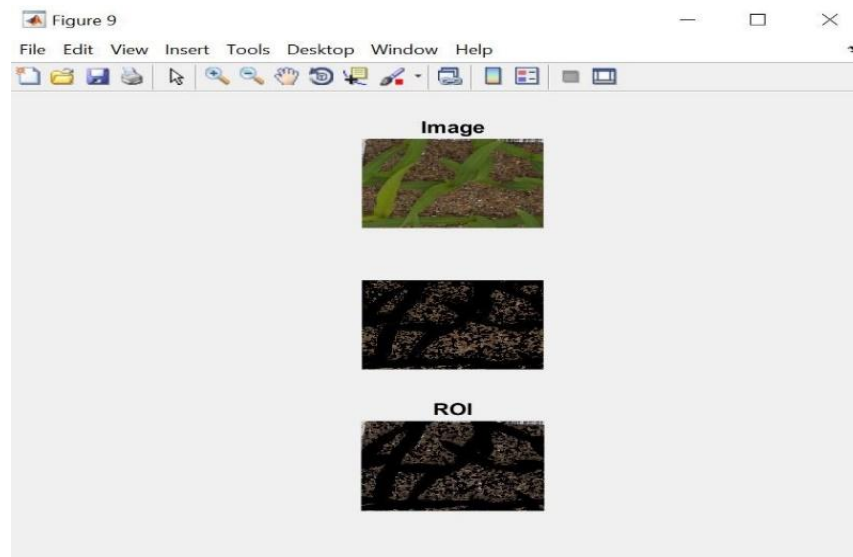

Fig 9: Region of Interest

[3] Feature Extraction: In picture preparing highlight determination is also alluded to as factor choice, or variable subset choice, it's the technique for picking an important element to be utilized in model development. include extraction might be an extraordinary kind of dimensionality decrease.

\section{Published By:}

Blue Eyes Intelligence Engineering \& Sciences Publication

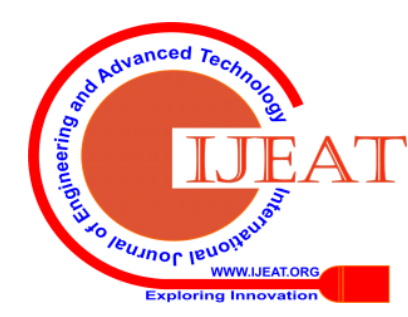


At the point when the information record to a calculation is simply too huge to possibly be prepared, at that point the info document will be changed into a diminished portrayal set of highlights. Changing the info record into the arrangement of highlights is named include extraction. Highlight extraction, is utilized to remove the individual cells from the information picture. We start GLCM by changing over the ROI's into grayscale, at that point we get the grayscale cooccurance framework. details = graycoprops(g,properties) figures the insights indicated in properties from the dim level co-event lattice g. g is a m-by-n-by-p exhibit of legitimate dim level co-event frameworks. On the off chance that $g$ is a variety of GLCMs, details is a variety of insights for each glcm. Next, we extricate data from details exhibit about Contrast, Correlation, Energy, Homogenity, Mean, Standard Deviation, Entropy, RMS, Variance, Smoothness, Kurtosis and Skewness.

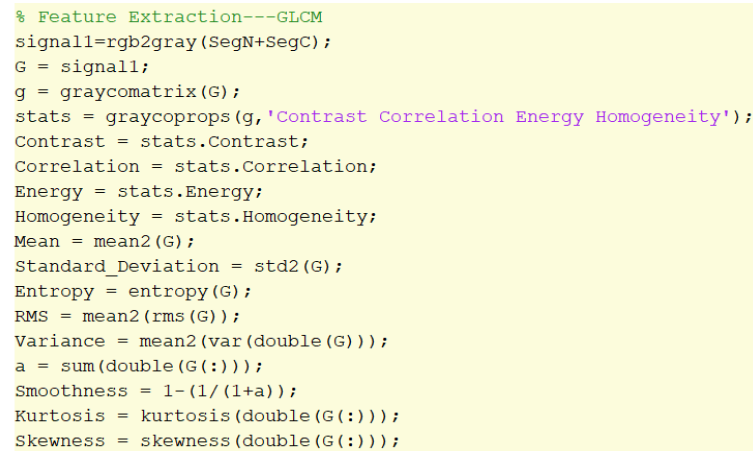

Inverse Difference Moment quantifies the neighbourhood homogeneity of an image. The frequency of co-event of pixel sets is upgraded once they are attracted dim worth and, in this manner, builds the IDM esteem.

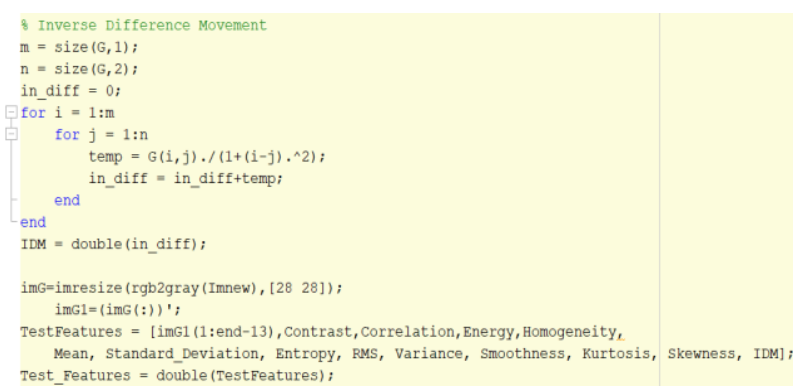

[4] Classification: Classification is to relate the satisfactory class mark (kind of surface) with the biopsy test by utilizing the estimations. The determination of noticeable highlights assumes a significant job in decreasing the computational unpredictability of a classifier.

[4.1] Convolutional Neural Network (CNN): In neural systems, Convolutional neural system (ConvNets or CNNs) is one among the most classifications to attempt to pictures acknowledgment, pictures arrangements. Items location, acknowledgment faces and so forth. In our venture CNN is utilized as a Classifier. CNN picture orders take an info picture, process it and group it under specific classes (Leukemia or Myeloma). In view of the picture goals, a picture is isolated into cluster of lattice of grayscale picture. [4.1.1] Convolution Layer: Convolution is that the main layer to separate highlights from an info picture. Convolution protects the association between pixels by learning picture highlights utilizing little squares of info information. It is a scientific procedure that takes two sources of info like picture network and a channel or piece. Convolution of an image with various channels can perform activities like edge location, obscure and hone by applying channels.

[4.1.2] Padding: Some of the time channel doesn't fit entirely fit the information picture. We have two alternatives:

1. Pad the image with zeros (zero-cushioning) so it fits.

2. Drop the piece of the picture where the channel didn't fit. This is called legitimate cushioning which keeps just substantial a piece of the picture.

[4.1.3] Non-Linearity (ReLU): ReLU's motivation is to present non-linearity in our ConvNet. Since, this present reality information would need our ConvNet to discover would be non-negative straight qualities. There are other non-direct capacities like tanh or sigmoid additionally can be utilized instead of ReLU. The greater part of the data researchers use ReLU since execution shrewd ReLU is best than other two.

[4.1.4] Pooling Layer: Pooling layers area would downsize the quantity of parameters when the photos are overlarge. Spatial pooling likewise called subsampling or down examining which lessens the dimensionality of each guide yet holds the significant data. Spatial pooling can be of various kinds:

- Max Pooling

- Average Pooling

- Sum Pooling

Max pooling take the most significant component from the amended element map. Taking the most significant component could likewise take the run of the mill pooling. Total of all components inside the element map call as total pooling.

[4.1.5] Fully Connected Layer: The layer we call as FC layer, we leveled our lattice into vector and feed it into a completely associated layer like neural system.

[5] Novelty: The growing technology plays a major role and techniques like Machine Learning; Deep Learning are used. A captured image undergoes processes like pre-processing and segmentation. K-means clustering is used for segmentation. After segmentation, they undergo classification using Machine learning algorithms. This process will help to identify the crop name with stages of the crop and then the accuracy of the system using CNN will be identified.

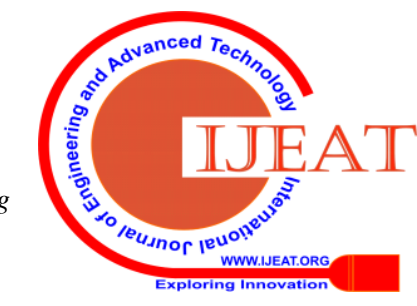




\section{Prediction of Crop and Weed Growth Stages using Neural Network in Machine Learning}

\section{RESULTS}
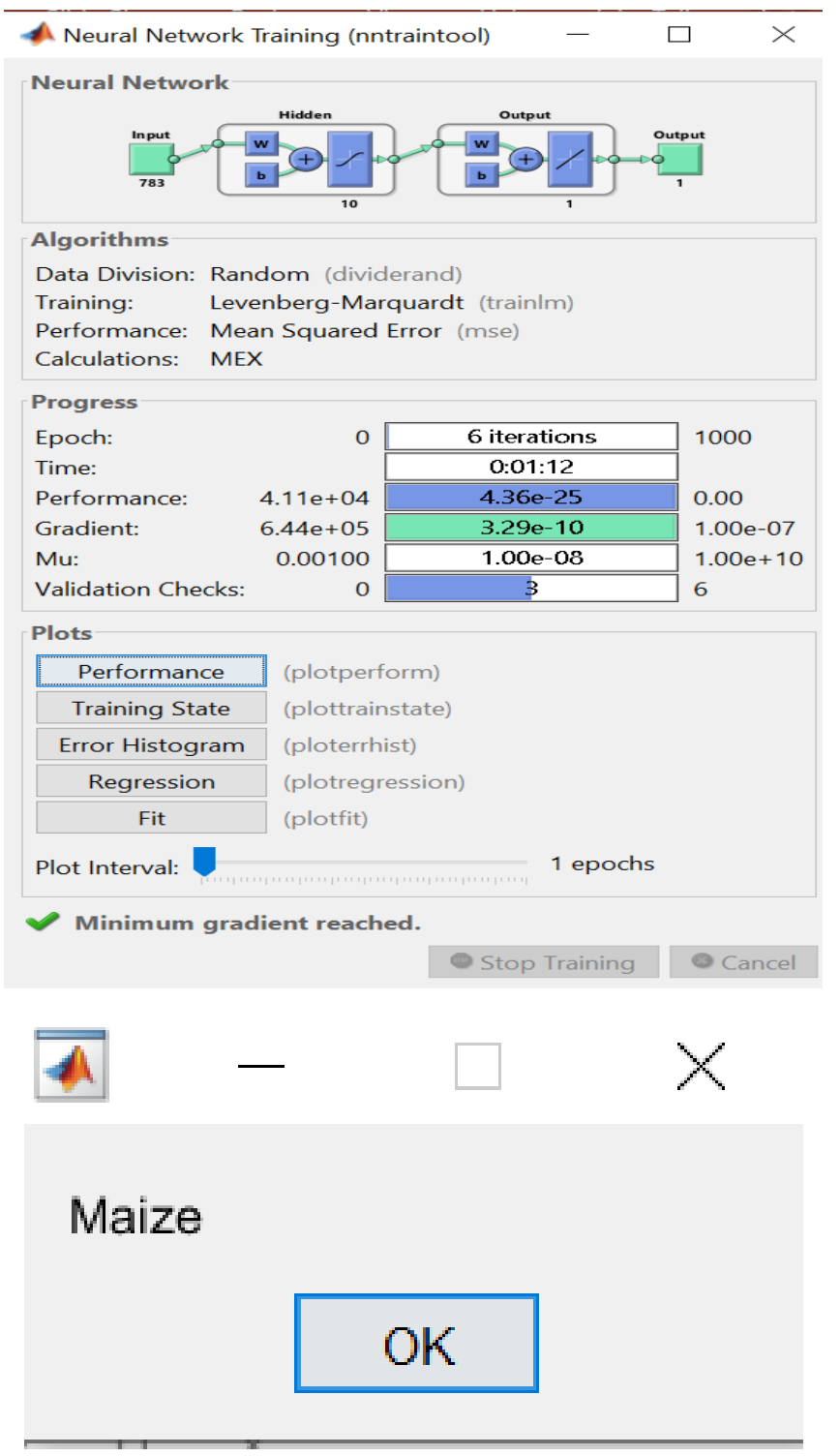

Fig 11: Crop Detected

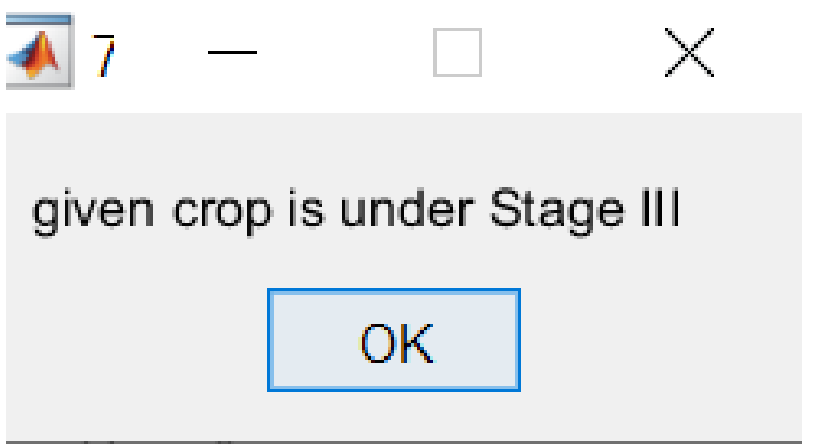

Fig 12: Stage Detected

In our Project, we had 11 plants to be classified into 3 stages each, where we had database of 350+ images with different angles and light intensities. The 11 plants are:

1.Black-grass 2. Charlock 3. Common Chickweed 4. Common wheat 5. Fat Hen 6. Loose Silky-bent 7. Maize 8. Scentless Mayweed 9. Shepherd's Purse 10. Small-flowered Cranesbill 11. Sugar beet Further we compared the accuracy of our algorithm with existing Random Forest algorithm as:

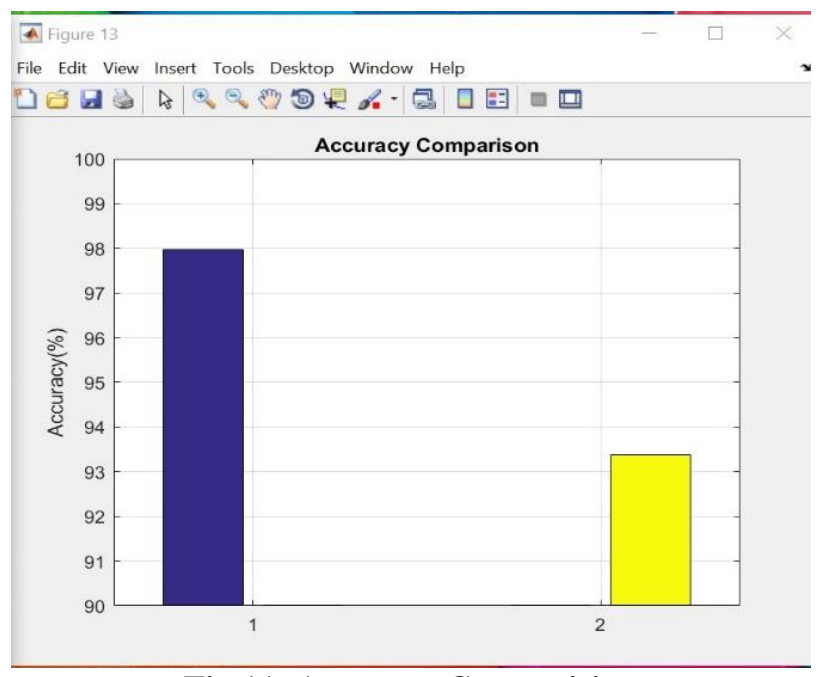

Fig 11: Accuracy Comparision

\section{CONCLUSION}

In this project, a convolutional neural system-based methodology has been applied for the characterization of various phenological phases of plants. Our CNN engineering can naturally group diverse phenological phases of eleven sorts of plants. So as to assess the exhibition and productivity of our profound learning-based methodology, a traditional AI approach dependent on physically removed highlights is likewise actualized. Textural highlights dependent on GLCM highlights have been extricated and joined arrangement of highlights are taken care of into an AI calculation. The characterization pace of the methodology dependent on physically extricated highlights are contrasted with those of our CNN based methodology. Trial results demonstrate that CNN put together methodology is altogether successful with respect to the eleven sorts of plants we probed. There are various ways profound learning can be applied on a dataset relying upon the size of the dataset. While a CNN can be prepared without any preparation, since it requires a huge measure of preparing information, an option has been finetuning pre-prepared CNN structures. There are many research bearings that we

\section{ACKNOWLEDGMENT}

We would like to express my sincere gratitude and heartfelt thanks to our project guide Dr. Jasmin Pemeena Priyadarsini $M$ who has been a constant pillar of motivation throughout the planning and implementation phases of this project. Her support and help, throughout the entire duration of the project, was of utmost importance in making it a success.

\section{REFERENCES}

1. Hulya Yalcin, "Plant Phenology Recognition using Deep Learning:Deep-Pheno", 6th International Conference on Agro-Geoinformatics, Agro-Geoinformatics, 2017

2. P. B. C. Leite, R. Q. Feitosa, A. R. Formaggio, G. A. O. P. Costa, K. Pakzad, I. D. A. Saches, "Crop type recognition based on Hidden Markov Models of plant phenology", XXI Brazilian Symposium on Computer Graphics and Image Processing, 2008

3. Fatih Gulac, Ulug Bayazit, "Plant and Phenology Recognition from Field Images Using Texture and Color Features", IEEE (SMC) International Conference on Innovations in Intelligent Systems and applications, INISTA, 2018

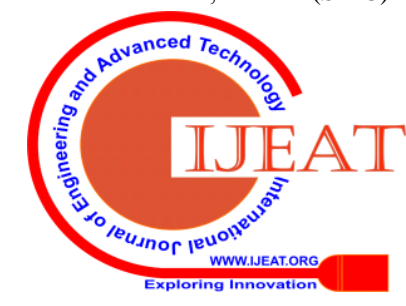


4. Fabio A. Faria, Jurandy Almeida, Bruna Alberton, Leonor Patricia C. Morellato, Anderson Rocha, Ricardo da S Torres, "Time series-based classifier fusion for fine-grained plant species recognition", Pattern Recognition Letters Vol. 81, 2016

5. DAWN M. BROWNING, THERESA M. CRIMMINS, DARREN K. JAMES, SHERI SPIEGAL, MATTHEW R. LEVI, JOHN P. ANDERSON, DEBRa P. C. PETERS, "Synchronous species responses reveal phenological guilds: implications for management", Ecosphere journal, SPECIAL FEATURE: DYNAMIC DESERTS, 2018

6. Jurandy Almeida, Jefersson A. dos Santos, Bruna Alberton, Leonor Patricia C. Morellato, Ricardo S. Torres, "Phenological visual rhythms: Compact representations for fine-grained plant species identification", Pattern Recognition Letters Vol.81, 2016, 90-100

7. Massimo Minervini, Andreas Fischbach, Hanno Scharr, Sotirios A. Tsaftaris, "Finely-grained annotated datasets for image-based plant phenotyping", Pattern Recognition Letters Vol.81, 2016, 80-89

\section{AUTHORS PROFILE}

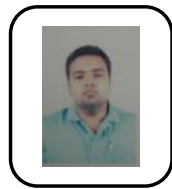

Devansh Dutt student of Vellore Institute of Technology. Vellore, India. He is currently pursuing his BTech in Electronics and Communication Engineering.

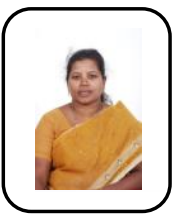

Dr. M.Jasmine Pemeena Priyadarsini obtained B.E. degree from Madras University in 1992 and M.E. degrees from Madurai Kamaraj University, Madurai in 1995.. She earned his Ph.D from Vellore Institute of Technology, Vellore, INDIA in 2014. She has published more than 45 research papers in National and International journals and reputed conferences. She has a teaching experience of about 25 years in Vellore Institute of Technology, Vellore in India. Presently, she is serving as Professor at Vellore Institute of Technology, India. She is a life member of Indian Society for Technical Education, IEEE society Membership, Fellow of Institution of Engineers, Fellow of Institution Electronics and Telecommunication Engineers. She has authored about four technical books. Her research areas include Digital Image Processing, Digital signal processing, Optical Signal Processing, Light wave Communication Systems, Optical Coding Theory and Biometric Image Processing. She is a reviewer of several international conferences and journals.

Smruti Ranjan Khuntia student of Vellore Institute of Technology. Vellore, India. He is currently pursuing his BTech in Electronics and Communication Engineering.

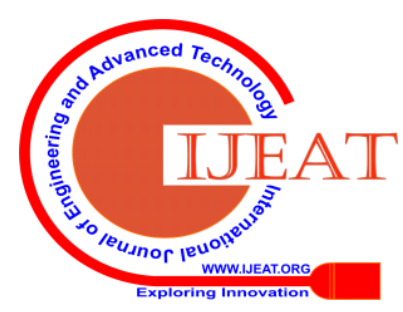

